\title{
Interfacial separation between elastic solids with randomly rough surfaces: comparison of experiment with theory
}

\author{
B. Lorenz and B.N.J. Persson \\ IFF, FZ-Jülich, D-52425 Jülich, Germany
}

\begin{abstract}
We study the average separation between an elastic solid and a hard solid with a nominal flat but randomly rough surface, as a function of the squeezing pressure. We present experimental results for a silicon rubber (PDMS) block with a flat surface squeezed against an asphalt road surface. The theory shows that an effective repulse pressure act between the surfaces of the form $p \sim \exp \left(-u / u_{0}\right)$, where $u$ is the average separation between the surfaces and $u_{0}$ a constant of order the root-mean-square roughness, in good agreement with the experimental results.
\end{abstract}

\section{Introduction}

Contact mechanics between solid surfaces is the basis for understanding many tribology processes $1,2,2,3,4,5$, 6, 7] such as friction, adhesion, wear and sealing. The two most important properties in contact mechanics are the area of real contact and the interfacial separation between the solid surfaces. For non-adhesive contact and small squeezing pressure, the (projected) contact area depends linearly on the squeezing pressure $8,0,10,11]$.

When two elastic solids with rough surfaces are squeezed together, the solids will in general not make contact everywhere in the apparent contact area, but only at a distribution of asperity contact spots. The separation $u(\mathbf{x})$ between the surfaces will vary in a nearly random way with the lateral coordinates $\mathbf{x}=(x, y)$ in the apparent contact area. When the applied squeezing pressure increases, the average surface separation $u=\langle u(\mathbf{x})\rangle$ will decrease, but in most situations it is not possible to squeeze the solids into perfect contact corresponding to $u=0$. We have recently developed a theory which predicts that, for randomly rough surfaces at low squeezing pressures, $p \sim \exp \left(-u / u_{0}\right)$, where the reference length $u_{0}$ depends on the nature of the surface roughness but is independent of $p[1,12]$. Here we will present experimental results to test the theory predictions [13]. We study the squeezing of a rubber block against an asphalt road surface. This topic is also important in the context of the air-pumping contribution to tire noise 14]. Thus the compression and outward flow of the air between a tread block and the road surface cavities during driving contribute to tire noise, similarly to how sound is generated during applause. A similar effect (but now involving decompression and inward flow of air) occur when a tread block leave the tire-road contact area.

\section{Theory}

We consider the frictionless contact between an elastic solid (elastic modulus $E$ and Poisson ratio $\nu$ ) with a flat surface and a rigid, randomly rough surface with the surface height profile $z=h(\mathbf{x})$. The separation between the average surface plane of the block and the average surface plane of the substrate (see Fig. 11) is denoted by $u$ with $u \geq 0$. When the applied squeezing force $p$ increases,

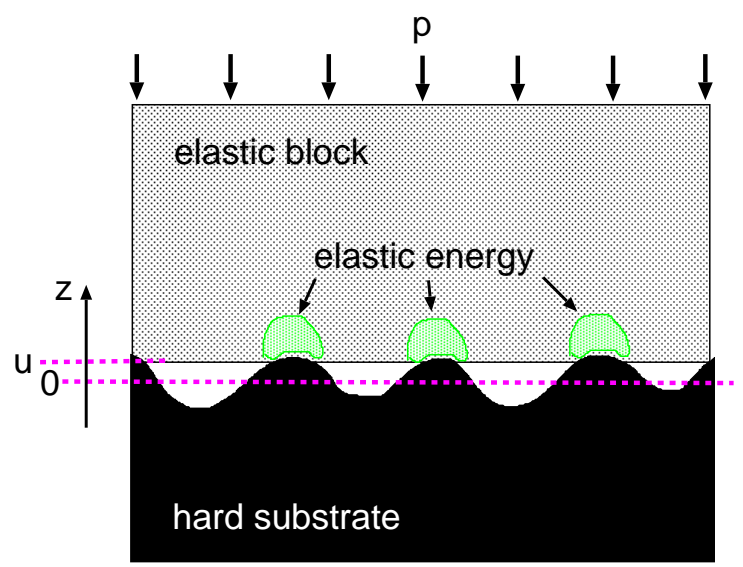

FIG. 1: An elastic block squeezed against a rigid rough substrate. The separation between the average plane of the substrate and the average plane of the lower surface of the block is denoted by $u$. Elastic energy is stored in the block in the vicinity of the asperity contact regions.

the separation between the surfaces at the interface will decrease, and we can consider $p=p(u)$ as a function of $u$. The elastic energy $U_{\mathrm{el}}(u)$ stored in the substrate asperity-elastic block contact regions must be equal to the work done by the external pressure $p$ in displacing the lower surface of the block towards the substrate. Thus,

$$
p(u)=-\frac{1}{A_{0}} \frac{d U_{\mathrm{el}}}{d u}
$$

where $A_{0}$ is the nominal contact area. Eq. (1) is exact 12, 15]. Theory shows that for low squeezing pressure, the area of real contact $A$ varies linearly with the squeezing force $p A_{0}$, and that the interfacial stress distribution, and the size-distribution of contact spots, are independent of the squeezing pressure 16]. That is, with increasing $p$ existing contact areas grow and new contact areas form in such a way that in the thermodynamic limit (infinite-sized system) the quantities referred to above remain unchanged. It follows immediately that for small load the elastic energy stored in the asperity contact region will increase linearly with the load, i.e., $U_{\mathrm{el}}(u)=u_{0} A_{0} p(u)$, where $u_{0}$ is a characteristic length 
which depends on the surface roughness (see below) but is independent of the squeezing pressure $p$. Thus, for small pressures (1) takes the form

$$
p(u)=-u_{0} \frac{d p}{d u}
$$

or 17

$$
p(u) \sim e^{-u / u_{0}} .
$$

To quantitatively derive the relation $p(u)$ we need an analytical expression for the asperity induced elastic energy. Within the contact mechanics approach of Persson we have 16, 18, 19]

$$
U_{\mathrm{el}} \approx A_{0} E^{*} \frac{\pi}{2} \int_{q_{0}}^{q_{1}} d q q^{2} P(q, p) C(q),
$$

where $E^{*}=E /\left(1-\nu^{2}\right)$ and where $P(q, p)=A(\zeta) / A_{0}$ is the relative contact area when the interface is studied at the magnification $\zeta=q / q_{0}$, which depends on the applied pressure $p$. The surface roughness power spectrum[16]

$$
C(q)=\frac{1}{(2 \pi)^{2}} \int d^{2} x\left\langle h(\mathbf{x} h(\mathbf{0})\rangle e^{-i \mathbf{q} \cdot \mathbf{x}},\right.
$$

where $\langle.$.$\rangle stands for ensemble average. Note that for$ complete contact $P=1$ and in this limit (3) is exact. For self affine fractal surfaces the prediction of the contact mechanics theory of Persson has been compared to numerical simulations 19, 20]. The numerical studies indicate that as the fractal dimension of the surface approaches 2 the Persson theory may become exact, while a small difference between theory and simulations are observed for larger fractal dimension 21]. Below we will compare the theory predictions to experimental data for an asphalt road surface which is fractal-like with the fractal dimension $D_{\mathrm{f}} \approx 2$. We find nearly perfect agreement between theory and experiment (see below), supporting the picture gained before based on numerical simulations.

Substituting (3) in (1) gives for small squeezing pressures [12]:

$$
p=\beta E^{*} e^{-u / u_{0}}
$$

For self affine fractal surfaces, the length $u_{0}$ and the parameter $\beta$ depend on the Hurst exponent $H$ and on $q_{0}$ and $q_{1}$. Most surfaces which are self affine fractal have the Hurst exponent $H>0.5$ (or the fractal dimension $\left.D_{\mathrm{f}}<2.5\right)$. For such surfaces $u_{0}$ and $\beta$ are nearly independent of the highest surface roughness wavevector, $q_{1}$, included in the analysis. For the substrate surface studied below we obtain from the measured surface roughness power spectrum (see Fig. 77) $u_{0}=0.30 \mathrm{~mm}$ and $\beta=0.59$. Note that $u_{0}$ is of order the root-mean-square roughness amplitude $\left(h_{\mathrm{rms}} \approx 0.29 \mathrm{~mm}\right.$ in the present case, see below).

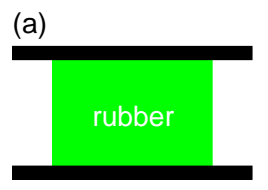

undeformed

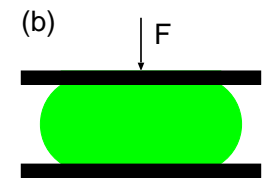

no slip

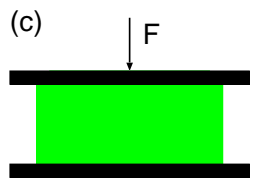

perfect slip
FIG. 2: A rubber block between two flat and rigid solid plates. (a) Undeformed state. (b) Squeezed block assuming no slip (i.e., high enough static friction) at the rubber-plate interfaces. (c) Squeezed block assuming perfect slip (i.e., no friction) at the rubber-plate interfaces.

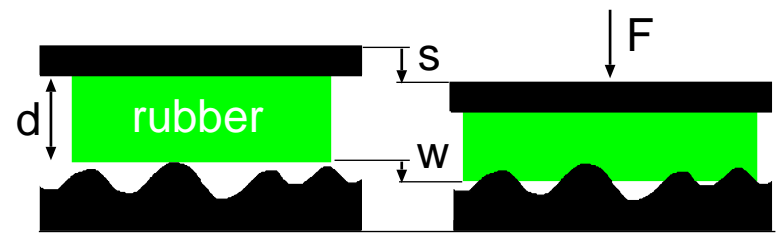

FIG. 3: A rubber block in contact with a rigid, randomly rough substrate. Left: no applied load. Right: The rubber block is squeezed against the substrate with the force $F$. The upper and the (average position of) the lower surface of the rubber block moves downwards by the distances $s$ and $w$, respectively. We assume perfect interfacial slip (no friction).

Consider a rubber block (elastic modulus $E$ ) with a flat surface (area $A_{0}$ ) and the thickness $d$. We will study both dry and lubricated interfaces (see Fig. 2) resulting in no slip and perfect slip at the two rubber-confining wall interfaces. If the block is squeezed against a rigid, randomly rough counter surface, the upper surface of the rubber block will move downwards by the distance $s$ (see Fig. 3), which is the sum of a uniform compression of the rubber block, $d \sigma / E$, and a movement (or penetration) $w$ of the average position of the lower surface of the rubber block into the valleys or cavities of the countersurface:

$$
s=w+d \sigma / E
$$

If $u$ denote the average separation between the block and the substrate (so that $u=0$ correspond to perfect contact) then

$$
w=h_{\max }-u
$$

where we have assumed that the initial position of the lower surface of the block correspond to the separation where the block just makes contact with the highest substrate asperity (as in Fig. 3, left), which is located a distance $h_{\max }$ above the average substrate surface plane. Using (4) we get

$$
\log (\sigma / E)=\log (4 \beta / 3)-u / u_{0}
$$

where $\sigma=F / A_{0}$ the squeezing pressure. Here we have used that $E^{*} / E=1 /\left(1-\nu^{2}\right) \approx 4 / 3$ since for rubber 
$\nu \approx 1 / 2$. Combining (5) and (6) gives

$$
u=h_{\max }-s+d \sigma / E
$$

Substituting this in (7) gives

$$
\log \left(\frac{\sigma}{E}\right)=\log \left(\frac{4 \beta}{3}\right)-\frac{1}{u_{0}}\left(h_{\max }-s+d \frac{\sigma}{E}\right)
$$

or

$$
\log \left(\frac{\sigma}{E}\right)=B+\frac{1}{u_{0}}\left(s-d \frac{\sigma}{E}\right)
$$

where $B=\log (4 \beta / 3)-h_{\max } / u_{0}$.

For no-slip boundary condition, Eq. (5) is replaced by

$$
s=w+d \sigma / E^{\prime}
$$

where the effective modulus $E^{\prime}>E$. Thus, in this case (8) takes the form

$$
\log \left(\frac{\sigma}{E^{\prime}}\right)=B^{\prime}+\frac{1}{u_{0}}\left(s-d \frac{\sigma}{E^{\prime}}\right)
$$

where $B^{\prime}=\log \left(4 \beta E / 3 E^{\prime}\right)-h_{\max } / u_{0}$.

\section{Experimental}

To test the theory presented above, we have performed the experiment indicated in Fig. 3. A rubber block with a flat surface was squeezed against an asphalt road surface. The displacement $s$ of the upper surface of the rubber block was changed in steps of $0.05 \mathrm{~mm}$, and the force $F$ was measured. For the experiment we used a test stand produced by SAUTER GmbH (Albstadt, Germany), normally used to measure spring constants. Using this test stand, we are able to measure forces up to $500 \mathrm{~N}$, and displacement with the resolution $0.01 \mathrm{~mm}$.

The rubber block was made from a silicone elastomer (PDMS). The PDMS samples were prepared using a twocomponent kit (Sylgard 184) purchased from Dow Corning (Midland, MI). This kit consists of a base (vinylterminated polydimethylsiloxane) and a curing agent (methylhydrosiloxane-dimethylsiloxane copolymer) with a suitable catalyst. From these two components we prepared a mixture of 10:1 (base/cross linker) in weight. The mixture was degassed to remove the trapped air induced by stirring from the mixing process and then poured into cylindric casts (diameter $D=3 \mathrm{~cm}$ and height $d=1 \mathrm{~cm}$ ). The bottom of these casts were made from glass to obtain smooth surfaces (negligible roughness). The samples were cured in an oven at $80{ }^{\circ} \mathrm{C}$ for over 12 hours.

The road surface used in this experiment was provided by Pirelli (Italian tire manufacturer). The topography was measured with contact-less optical methods using a chromatic sensor with two different optics produced by Fries Research \& Technology GmbH (Bergisch Gladbach, Germany). To identify the elastic modulus $E$, the PDMS sample was first squeezed against a smooth substrate in

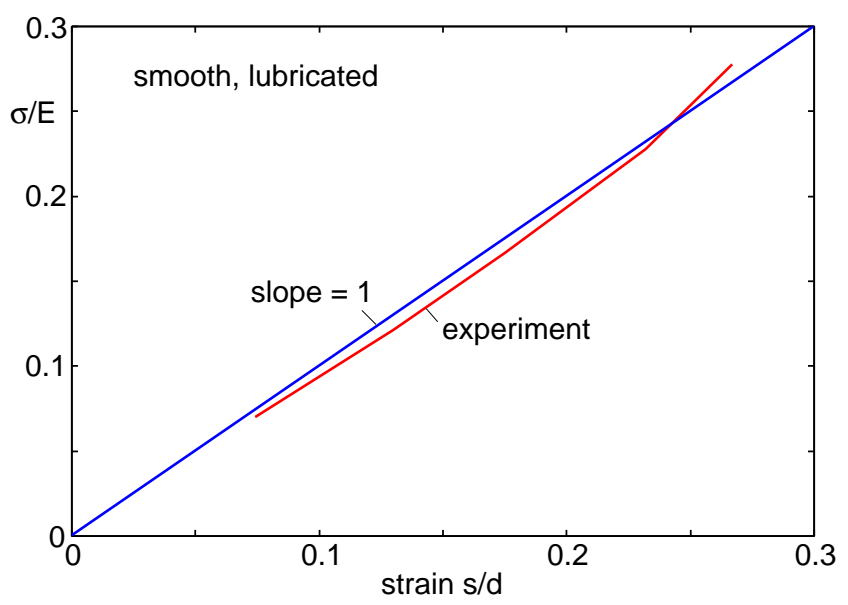

FIG. 4: The stress $\sigma$ (in units of the elastic modulus $E$ ) as a function of the strain $s / d$, where $s$ is the displacement of the upper surface and $d$ the thickness of the block. In the calculation we used $E=2.3 \mathrm{MPa}$. For a PDMS rubber block confined between two smooth lubricated (wet) surfaces.

a compression test. We measured the force $F$ over the displacement $s$ for two different cases. First there was no lubrication used and the PDMS sample deformed laterally at the force-free area as shown in Fig. 2(b), because no slip occurred at the contact areas. Second we lubricated the contact areas to obtain perfect slip at the interfaces (see Fig. 2(c)). We used polyfluoroalkylsiloxane (PFAS), a fluorinated silicone oil supplied by ABCR GmbH \& Co. KG (Karlsruhe, Germany). Because of its high viscosity $(\eta=1000 \mathrm{cSt})$, the fluid is an excellent lubricant also under extreme pressure applications and should therefore not easily be squeezed out of the contact area. Also it does not react (or interdiffuse) with the PDMS elastomer.

\section{Results}

Consider first flat surfaces. In Fig. 4 we show the measured relation between the stress and the strain for lubricated surfaces (so that the shear stress vanish on the boundaries). If the stress is normalized with $E=$ $2.3 \mathrm{MPa}$ a nearly strait line with the slope 1 will result so that the relation $\sigma=E s / d$ holds. The elastic modulus $E=2.3 \mathrm{MPa}$ is consistent with the elastic modulus reported in the literature for similar silicon rubbers [22].

We have also performed experiments for dry surfaces. In this case no (or negligible) slip occur at the interface with the confining walls, and visual inspection of the system showed that the rubber bulge laterally at the force-free area (see Fig. 2(b)). We still expect a linear (or near linear) relation between stress and strain but the effective elastic modulus $E^{\prime}$ is larger than for lubricated interfaces. Thus, the effective elastic modulus deduced from the experimental data (see Fig. 5) $E^{\prime} \approx 4.2 \mathrm{MPa}$ is about $80 \%$ larger than for the lubricated interface. To 


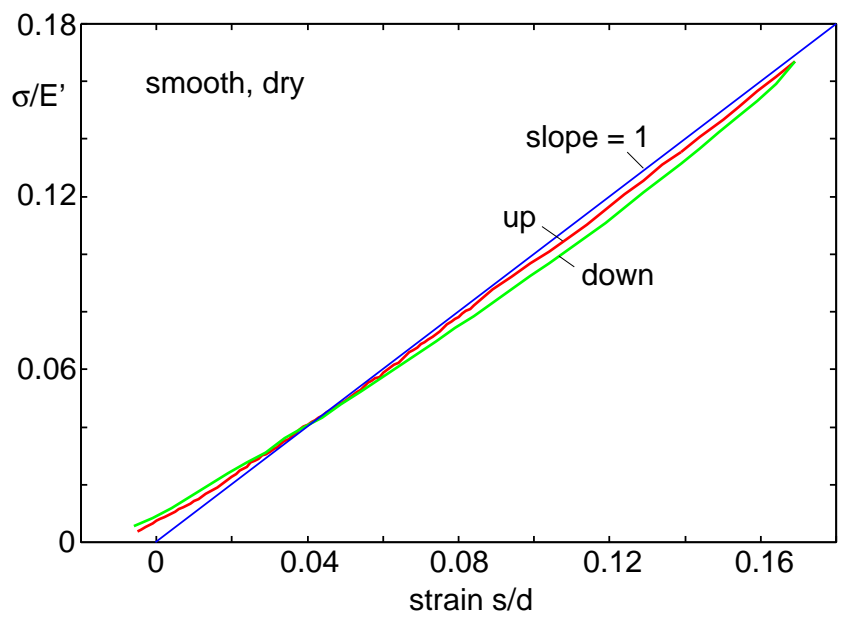

FIG. 5: The stress $\sigma$ (in units of the elastic modulus $E^{\prime}$ ) as a function of the strain $s / d$, where $s$ is the displacement of the upper surface and $d$ the thickness of the block. In the calculation we used the effective modulus $E^{\prime}=4.2 \mathrm{MPa}$. For a PDMS rubber block confined between smooth dry surfaces. The two experimental curves corresponds to increasing and decreasing the strain.

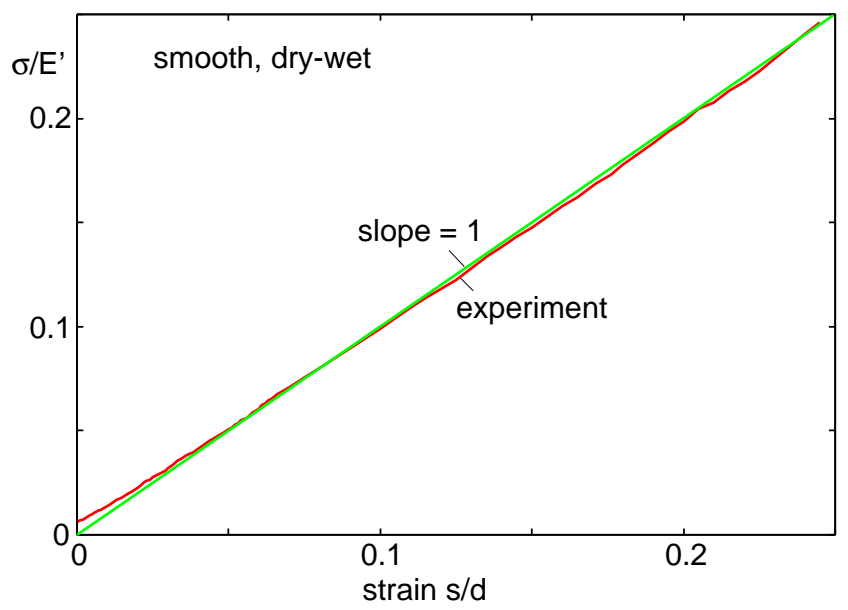

FIG. 6: The stress $\sigma$ (in units of the elastic modulus $E^{\prime}$ ) as a function of the strain $s / d$, where $s$ is the displacement of the upper surface and $d$ the thickness of the block. In the calculation we used the effective modulus $E^{\prime}=2.9 \mathrm{MPa}$. For a PDMS rubber block confined between one lubricated (wet) surface and one dry surface.

check the measuring system for hysteresis effects, some of the experiments were performed bidirectional. The results are shown in Fig. 5 where the strain was increased and after that slowly decreased again. Negligible hysteresis occur, as expected because of the low glass transition temperature of PDMS.

The increase in the effective elastic modulus in compression, from 2.3 $\mathrm{MPa}$ to $4.2 \mathrm{MPa}$, when going from slip to no-slip boundary condition, is consistent with the prediction of the Lindley equation [23], which in the present

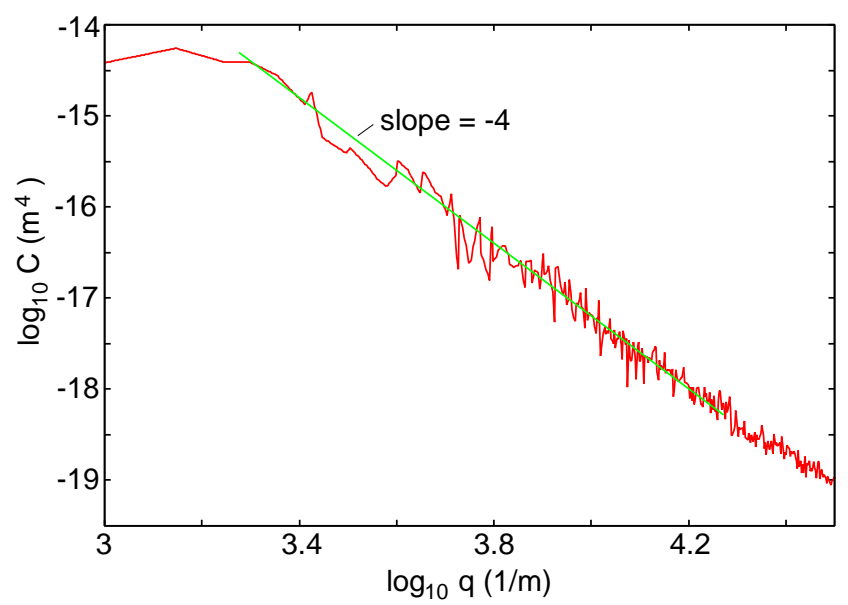

FIG. 7: The surface roughness power spectrum $C$, as a function of the wavevector $q$ (log-log scale), for an asphalt road surface. The strait green line has the slope -4 , corresponding to the Hurst exponent $H=1$ (fractal dimension $D_{\mathrm{f}}=2$ ).

case takes the form

$$
E^{\prime} \approx E\left(1+1.4 S^{2}\right)
$$

For a cylinder the shape factor $S=R / 2 d$. In the present case $E=2.3 \mathrm{MPa}$ and $S=0.75$ giving $E^{\prime}=4.1 \mathrm{MPa}$ which agree very well with the measured value (4.2 MPa).

We have also studied the case where one surface is lubricated and the other dry. In this case the the rubber will displace laterally in an assymetric way (as in Fig. 10(b)) and the measured effective elastic modulus $E^{\prime}=2.9 \mathrm{MPa}$ (see Fig. 6), is slightly smaller than the the average of the effective $E$-modulus obtained assuming no-slip and complete slip on both surfaces: $(2.3+4.2) / 2 \mathrm{MPa} \approx 3.3 \mathrm{MPa}$.

We will now present experimental results for a rubber block squeezed against an asphalt road surface. The surface roughness power spectrum of the road surface is shown in Fig. 7. The surface has the root-mean-square roughness $h_{\mathrm{rms}} \approx 0.29 \mathrm{~mm}$, and for the wave vector $q>q_{0} \approx 2500 \mathrm{~m}^{-1}$ it is (on a log-log scale) well approximated by a strait line with the slope corresponding to a self-affine fractal surface with the fractal dimension $D_{\mathrm{f}}=2$. For $q<q_{0}, C(q)$ is approximately constant; we refer to $q_{0}$ as the roll-off wavevector.

In Fig. 8 we show the natural logarithm of the squeezing pressure (divided by the effective elastic modulus) as a function of $s-d \sigma / E^{\prime}$, where $s$ is the displacement of the upper surface of the rubber block relative to the substrate, and where $d$ is the thickness of the rubber block. In the calculation we used the effective elastic modulus $E^{\prime}=4.8 \mathrm{MPa}$ and $B^{\prime}=-6.85$. The value of $B^{\prime}$ has been calculated using (9) (using the measured $h_{\max }$ ) so that the only fitting parameter is the effective elastic modulus $E^{\prime}$, which however agree rather well with the measurements for flat surfaces $\left(E^{\prime}=4.2 \mathrm{MPa}\right)$. 


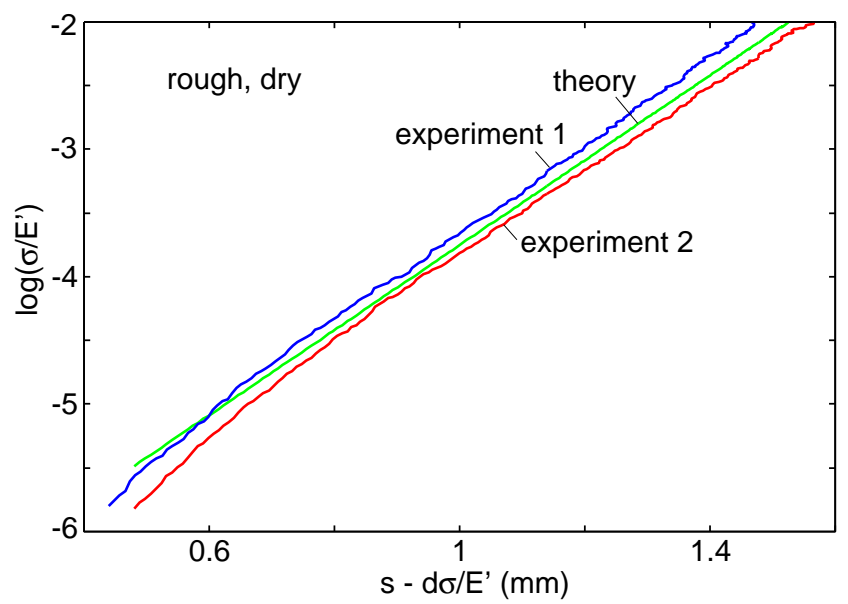

FIG. 8: The natural logarithm of the squeezing pressure (divided by the effective elastic modulus) as a function of $s-d \sigma / E^{\prime}$, where $s$ is the displacement of the upper surface of the rubber block relative to the substrate, and where $d$ is the thickness of the rubber block. In the calculation we used the effective elastic modulus $E^{\prime}=4.8 \mathrm{MPa}$ and $B^{\prime}=-6.85$. For dry contact.

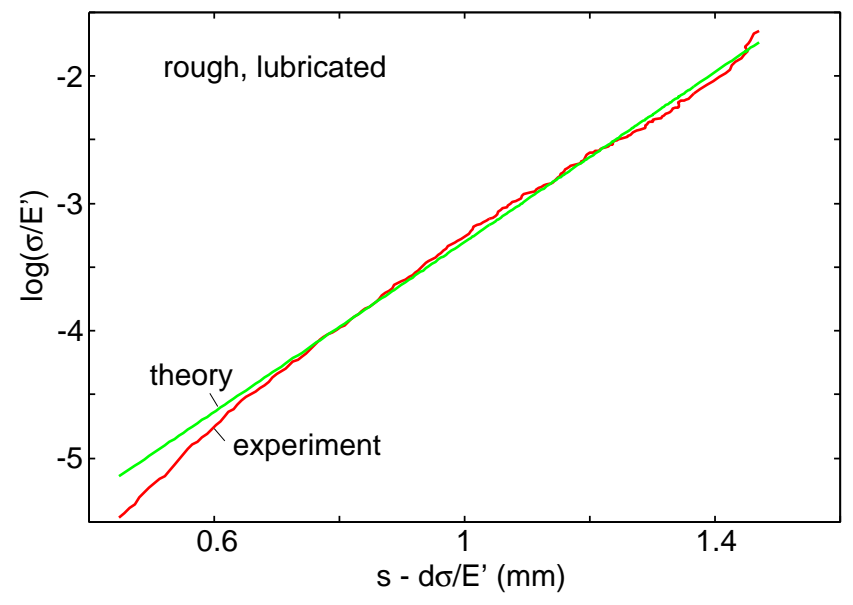

FIG. 9: The natural logarithm of the squeezing pressure (divided by the effective elastic modulus) as a function of $s-d \sigma / E^{\prime}$, where $s$ is the displacement of the upper surface of the rubber block relative to the substrate, and where $d$ is the thickness of the rubber block. For lubricated (wet) contact. In the calculation we used the effective elastic modulus $E^{\prime}=3.4 \mathrm{MPa}$ and $B^{\prime}=-6.5$.

In Fig. 9 we show the same as in Fig. 8 but now for lubricated surfaces. In the calculation we used the effective elastic modulus $E^{\prime}=3.4 \mathrm{MPa}$ and $B^{\prime}=-6.50$. Note that this value for $B^{\prime}$ is slightly smaller than for dry contacts. The difference $\Delta B^{\prime}=-6.50-(-6.85)=0.35$ just reflect the difference in the effective $E$-modulus since according to (9) $\Delta B^{\prime}=\log \left[E^{\prime}(\right.$ dry $) / E^{\prime}$ (lubricated) $]=$ $\log (4.8 / 3.4) \approx 0.35$. The $E^{\prime}$ value is larger than the $E$-modulus measured for flat lubricated surfaces $(E=$ 2.3 $\mathrm{MPa}$ ), but this can be understood as follows.
Visual inspection of the contact between the rubber cylinder and the two confining walls shows that, as expected from above, the rubber block slip against the top (flat) steel surface, while no slip (or only very limited slip) occur against the rough substrate surface, see Fig. 10(b). This is consistent with the fact that the observed elastic modulus is larger than $E=2.3 \mathrm{MPa}$, as obtained above when complete slip occur at both (lubricated) surfaces. In fact, the observed effective $E$-modulus $(3.4 \mathrm{MPa})$ is rather close to the value $2.9 \mathrm{MPa}$ measured for smooth surfaces when slip occur at one surface and no slip at the other surface. The fact that no (or very small) slip occur at the interface between the rubber and the rough substrate surface may be due to at least two facts:

1) The pressure in the asperity contact regions are much higher than the average pressure, and the asperity contact regions much smaller than the nominal contact area, resulting in much faster squeeze-out of the lubricant oil from the asperity contact regions, as compared to the case of flat surfaces, and consequently to higher friction in the contact regions.

2) The substrate surface roughness on different length scales contribute to the friction during slip because of the viscoelastic deformations of the rubber on different length scales. However, since for silicon rubber viscoelastic dissipation only occur at very high frequencies, it is likely that this effect is small in the present case.

The measured $E^{\prime}$-values for rough surfaces $(4.8 \mathrm{MPa}$ and $3.4 \mathrm{MPa}$ ) are roughly $14 \%$ larger than for smooth surfaces (4.2 $\mathrm{MPa}$ and 2.9 $\mathrm{MPa}$ ), as obtained assuming no-slip on the confining surfaces in one case, and slip on only one of the confining surfaces in the other case. The origin of this (small) difference in effective elastic modulus is not known to us.

Finally, we note that for $s-d \sigma / E^{\prime}<0.6 \mathrm{~mm}$ the experimental curve in Fig. 9 drops of faster with decreasing interfacial separation than predicted by the theory. (The same effect can also be seen in Fig. 8 and has also been observed in molecular dynamics calculations [15].) This is a finite size effect: The theory is for an infinite system which has (arbitrary many) arbitrary high asperities, and contact between the two solids will occur for arbitrary large surface separation, and the relation $p \sim \exp \left(-u / u_{0}\right)$ holds for arbitrary large $u$. On the other hand a finite system has asperities with height below some finite length $h_{\max }$, and for $u>h_{\max }$ no contact occur between the solids and $p=0$.

\section{Summary and conclusion}

We have presented a combined experimentaltheoretical study of the contact between a rigid solid with a randomly rough surface and an elastic block with a flat surface. The interfacial separation as a function of the squeezing pressure has been derived theoretically and has been compared to the experimental results. We find nearly perfect agreement between theory and experimen- 
(a)

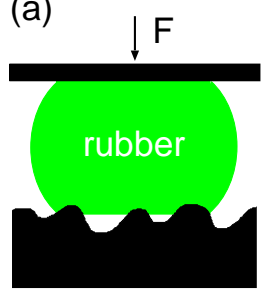

(b)

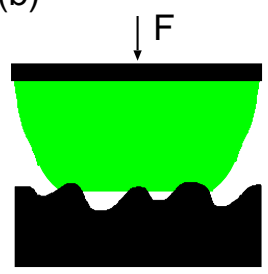

FIG. 10: A rubber block squeezed between a rigid solid plate and a rigid randomly rough substrate. (a) Dry surfaces and (b) lubricated surfaces.

tal data for an asphalt road surface. We conclude that for non-adhesive interaction and small applied pressure, $p \sim \exp \left(-u / u_{0}\right)$, where $p$ is the squeezing pressure and $u$ the average interfacial separation, and $u_{0}$ a constant of order the root-mean-square roughness of the combined surface profile. In addition, the experimental results indicate that for surfaces with fractal-like roughness profiles the Persson contact mechanics theory may be exact for the fractal dimension $D_{\mathrm{f}}=2$. We plan to extend the study above to surfaces with other fractal dimension to test the theory in more general cases. The presented results may be of great importance for, e.g., heat transfer, lubrication, sealing, optical interference, and tire noise related to air-pumping.

\section{Acknowledgments}

The authors would like to thank Pirelli Pneumatici for support. We thank O.D. Gordan (IBN, FZ-Jülich) for help with preparing the PDMS rubber blocks.

[1] M. Benz, K.J. Rosenberg, E.J. Kramer and J.N. Israelachvili, J. Phys. Chem B110, 11884 (2006).

[2] B.N.J. Persson and C. Yang, J. Phys.: Condens. Matt. 20, 315011 (2008)

[3] B.N.J. Persson, J. Phys.: Condens. Matt. 20, 315007 (2008)

[4] F.P. Bowden and D. Tabor, Friction and Lubrication of Solids (Wiley, New York, 1956).

[5] K.L. Johnson, Contact Mechanics, (Cambridge University Press, Cambridge, 1966).

[6] B.N.J. Persson, Sliding Friction: Physical Principles and Applications, 2nd edn. (Springer, Heidelberg, 2000).

[7] J.N. Israelachvili, Intermolecular and Surface Forces (Academic, London (1995)).

[8] B.N.J. Persson, J. Chem. Phys. 115, 3840 (2001).

[9] J.A. Greenwood and J.B.P. Williamson, Proc. Roy. Soc. London A295, 300 (1966).

[10] M.H. Müser, Phys. Rev. Lett. 100, 055504 (2008).

[11] G. Carbone and F. Bottiglione, J. Mech. Phys. Solids 56, 2555 (2008).

[12] B.N.J. Persson, Phys. Rev. Lett. 99, 125502 (2007)

[13] Experiments involving the squeezing of rubber blocks against rough surfaces have been performed by G. Gäbel and M. Kröger [proceedings of INTER-NOISE 2007, 2831 August 2007, Istanbul, Turkey] but without comparing the experimental results to theory.

[14] S. Kim, W. Jeong, Y. Park and S. Lee, The Journal of the Acoustical Society of America 119, 3799 (2006).

[15] C. Yang and B.N.J. Persson, J. Phys. Condens. Matter 20, 215214 (2008).

[16] B.N.J. Persson, Surface Science Reports 61, 201 (2006).

[17] We note that the result (2) differs drastically from the prediction of asperity contact mechanics theories such as those of the Bush et al [A.W. Bush, R.D. Gibson and T.R. Thomas, Wear 35, 87 (1975)] and the theory of Greenwood and Williamson [J.A. Greenwood and J.B.P. Williamson, Proc. Roy. Soc. London Ser. A295, 300 (1966)].

[18] B.N.J. Persson, Eur. Phys. J E8, 385 (2002).

[19] C. Campana, M.H. Müser and M.O. Robbins, J. Phys.: Condens. Matter 20, 354013 (2008).

[20] S. Hyun, L. Pei, J.F. Molinari and and M.O. Robbins, Phys. Rev. E70, 026117 (2004).

[21] B.N.J. Persson, J. Phys. Condens. Matter 20, 312001 (2008).

[22] See, e.g., Bongaerts et al. [J.H.H. Bongaerts, K. Fourtouni and J.R. Stokes, Tribology International 40, 1531 (2007)], where they report the Young's modulus $E=$ $2.4 \mathrm{MPa}$ for PDMS prepared in the way as in our case, using Sylgard 184 with a base/curing agent mass ratio 10:1. Similarly, Scheibert et al. [J. Scheibert, A. Prevost, J. Frelat, P. Rey and G. Debregeas, EPL 83, 34003 (2008)] obtain the Young's modulus $2.2 \pm 0.1 \mathrm{MPa}$.

[23] See, e.g., www.rubber-stichting.info/art2nr13.html 